\title{
Improved Adherence to Positive Airway Pressure Treatment after Covering National Health Insurance in Patient with Obstructive Sleep Apnea: A Tertiary Sleep Center Review
}

\author{
Hyo-Eun Yoon ${ }^{1}$, Chang-Jin Jeon ${ }^{2}$, Jaechun Hwang ${ }^{3}$, Ho-Won Lee ${ }^{3,4}$, Ji-Ye Jeon ${ }^{3}$ \\ ${ }^{1}$ Graduate School of Life Science, Kyungpook National University, Daegu, \\ ${ }^{2}$ Department of Biology, School of Life Sciences, College of Natural Sciences, and Brain Science and Engineering Institute, \\ Kyungpook National University, Daegu, \\ ${ }^{3}$ Department of Neurology, Kyungpook National University School of Medicine, Kyungpook National University Chilgok Hospital, Daegu, \\ ${ }^{4}$ Brain Science and Engineering Institute, Kyungpook National University, Daegu, Korea
}

Received November 2, 2020

Revised December 10, 2020

Accepted January 4, 2021

Address for correspondence

Ji-Ye Jeon, MD

Department of Neurology,

Kyungpook National University

School of Medicine,

Kyungpook National University

Chilgok Hospital,

807 Hoguk-ro, Buk-gu,

Daegu 41404, Korea

Tel: $+82-53-200-2168$

Fax: +83-53-200-2029

E-mail: jeonjiye87@gmail.com
Objectives: Obstructive sleep apnea (OSA) is a common clinical disease which has been associated with cardiovascular disease and sudden death. Positive airway pressure (PAP) is the most effective treatment for OSA; however, most patients experience difficulties in tolerating machines, masks, or economic burdens. In Korea, National Health Insurance (NHI) application for PAP started in July 2018. Therefore, this study aimed to evaluate the PAP adherence and factors contributing to good adherence after NHI coverage in Korea. Methods: A retrospective study was performed to examine 89 patients with OSA who were treated with PAP in 2019. All medical records were analyzed to obtain demographic and clinical data, including personal history, polysomnographic data, and PAP results. Results: A total of 66 participants were included, of which 56 (84.8\%) were maintained on PAP therapy after 3 months, and 32 of 42 (76.1\%) were maintained on PAP therapy after 6 months; prolonged rapid eye movement (REM) sleep latency, increased nonREM (NREM) apnea-hypopnea index (AHI), and supine AHI significantly contributed to good PAP adherence for 3 months. At 6 months, prolonged REM sleep latency, increased NREM AHI, supine AHI, and increased arousal index significantly contributed to good PAP adherence. However, comorbid hypertension contributed to poor PAP compliance. Conclusions: PAP adherence in patients with OSA was $84.8 \%$ after 3 months, and $76.1 \%$ after 6 months after NHI coverage in a regional tertiary sleep center in Korea. Prolonged REM sleep latency and increased supine AHI and NREM AHI significantly contributed to good adherence, whereas comorbid hypertension was a factor for poor PAP compliance.

J Sleep Med 2021;18(1):22-28

Key Words: Obstructive sleep apnea, Compliance, Positive-pressure respiration, National Health Insurance.

\section{Introduction}

Obstructive sleep apnea (OSA) is a common clinical disease, and the prevalence of OSA among middle-aged individuals is $2 \%$ in women and $4 \%$ in men. ${ }^{1}$ OSA is characterized by the cessation and reduction of nasal airflow during sleep, associated with blood oxygen desaturation. Clinical symptoms include snoring, frequent awakening, daytime fatigue, excessive daytime sleepiness, diminished intellectual ability, and changes in

This is an Open Access article distributed under the terms of the Creative Commons Attribution Non-Commercial License (https://creativecommons.org/licenses/by-nc/4.0) which permits unrestricted non-commercial use, distribution, and reproduction in any medium, provided the original work is properly cited. personality. During sleep apnea, blood oxygen levels decrease due to airway obstruction during sleep, and frequent awakening in response to reduced oxygen levels can activate the sympathetic nervous system, which is known to have adverse effects that contribute to the development of cardiovascular diseases. Overall, previous studies have shown that individuals with OSA have a 1.37-fold higher risk of developing hypertension than normal individuals. ${ }^{2}$ Individuals with apnea-hypopnea index (AHI) values greater than 5.3 per hour are 23 times as likely to develop myocardial infarction than those with lower AHI values. ${ }^{3}$ Individuals with severe OSA are 2.4 times more likely to develop congestive heart failure than those without 
apnea. ${ }^{4}$ The Wisconsin sleep cohort study found that mortality among sleep apnea patients increased significantly according to apnea severity, based on the results of an 18-year followup study. A 5.2-fold increase in cardiovascular mortality has also been reported for severe sleep apnea patients. ${ }^{5}$

Positive airway pressure (PAP) treatment is the first-choice treatment for most OSA patients, and in general, OSA treatments consist of lifestyle modifications, such as weight reduction and reducing the use of alcohol, sedatives, and sleep medications. PAP has been shown to reduce daytime sleepiness, oxygen desaturation, heart rate, and pulmonary pressure, improve cognitive performance, and increase quality of life. ${ }^{6}$ Despite improvements made in PAP technology, most patients continue to experience difficulties tolerating the machine, the mask or economic burden, and adherence to PAP treatment remains a clinically significant problem. ${ }^{7} \mathrm{PAP}$ adherence is generally determined by a number of factors, such as the medical, social and economic characteristics of the patient and the patient's understanding of the disease, in addition to side effects of the PAP.

Meantime, the cost of PAP therapy in Korea was about 2,000 dollars for the purchase of a machine, and if patients rent it, the monthly rental fee was around 250 dollars. Therefore, even though patient recognized the need for PAP, they often stopped treatment or missed the opportunity for treatment due to economic burden.

In Korea, there is a single universal health insurance system called National Health Insurance (NHI), which provides insurance for over $99 \%$ of the total population. ${ }^{8}$ Finally, NHI started to cover the costs of PAP treatment from July 2018. As a result, the rental fee of PAP has been dramatically reduced to 13-15 dollars monthly, which is about one-twentieth less than before. No place in the world has a public health insurance system that guarantees the whole nation and treated PAP as cheaply as Korea. Also studies focusing on economic burden in adherence of PAP treatment have rarely been conducted. Therefore, this study aimed to evaluate the PAP adherence and factors that affect adherence in patients with OSA since PAP treatment was included to NHI policy in Korea.

\section{Methods}

\section{Participants}

Participants with a history of snoring and/or apneas, who visited a sleep center at the Kyungpook National University Chilgok Hospital in Daegu, South Korea, between January 2019 and December 2019, were screened for this study, retrospectively. The participants in this study included aged between 20 and 85 years old. Those who were $<20$ years old and those who had previously used PAP within 6 weeks of enroll- ment were excluded. A total of 89 patients were examined using night polysomnography (PSG); however, 23 participants did not have sleep apnea and they were excluded from the study. Finally, 66 participants were prescribed PAP treatment. A total of 42 participants were treated with PAP for 6 months, and 66 participants were treated with PAP for 3 months, which was the minimum criteria to receive health insurance in Korea. We defined "good adherence" to PAP treatment as those patients who used PAP more than $70 \%$ of the monitoring periods, and average time of PAP usage per one night was more than 4 hours. This study was approved by the Institutional Review Board of Kyungpook National University (approval number: 2020-02-018).

\section{Polysomnography and PAP treatment}

Standard, full-night PSG techniques were recorded with Neuvo digital recording equipment (Compumedics, Australia), and the time required for inspection was at least 6 hours $30 \mathrm{~min}$ utes. Sleep stages were detailed according to standard electroencephalography, electrooculography, and electromyography (EMG) criteria by American Academy of Sleep Medicine scoring manuals. Apnea and hypopnea were recorded by an oronasal flow cannula, attached to a pneumotachograph. Arterial oxygen saturation was measured by pulse oximetry, using a finger probe. Thoracic and abdominal movements were recorded by using piezoelectric belts, to document the respiratory effort. Periodic limb movements were recorded using surface EMG electrodes on the tibialis anterior muscle of the lower extremity.

Obstructive apnea was defined by a decrease of more than approximately $90 \%$ of the baseline amplitude airflow for at least 10 seconds, in the presence of thoracoabdominal effort. Central apnea was determined if both airflow and respiratory efforts were absent for at least 10 seconds in duration, and mixed sleep apnea was diagnosed by the combination of both obstructive and central sleep apnea patterns. Hypopnea was scored if the magnitude of the ventilation signal decreased by more than approximately $30 \%$ of the baseline amplitude for at least 10 seconds and was associated with at least a 3\% drop in oxygen saturation, as measured by finger pulse oximetry, or accompanied arousals. ${ }^{9}$ OSA was diagnosed when AHI was 5 or more and there were weekly symptoms related to sleep apnea. The severity of OSA was classified as mild when AHI is 5 or more and less than 15 , moderate when it is 15 or more and less than 30 , and severe when it is 30 or more. PAP treatment was started to patient who was diagnosed with moderate OSA or more. Sixty of 66 patients were prescribed automatic PAP, and 6 out of 66 patients who agreed to re-examination of polysomnography for PAP titration were prescribed continuous PAP, and their average pressure was $8.3 \mathrm{~cm} \mathrm{H}_{2} \mathrm{O}$. 


\section{Data analysis}

Continuous variables were presented as the frequency, mean, and standard deviation. We also conducted a series of internal subgroup comparisons, using the Mann-Whitney U test. To determine the contributing factors associated with PAP adherence, data analysis was performed for score derivations, based on univariate and multivariate logistic regressions. A $p$-value $<0.05$ was considered significant. Statistical analyses were performed using IBM SPSS statistics version 21.0 (IBM Corp., Armonk, NY, USA).

\section{Results}

\section{Demographic characteristics}

For the total study period, 66 participants with sleep apnea were recruited (Table 1). The mean age of the participants was $55.7 \pm 13.5$ years. Fifty $(75.7 \%)$ were males and $16(24.2 \%)$ were females. The mean height was $166.8 \pm 9.4 \mathrm{~cm}$, the mean weight was $75.2 \pm 17.4 \mathrm{~kg}$, and the mean body mass index (BMI) was $26.9 \pm 5.4 \mathrm{~kg} / \mathrm{m}^{2}$. The mean snoring index value, defined as the number of detected snoring events per hour, estimated at 30-second intervals, was $336.4 \pm 188.6$. The mean AHI value was $35.2 \pm$ 20.3. The average peripheral oxygen saturation was $92.4 \pm 4.5 \%$.

\section{Adherence based on 6 months}

At the time of the survey, 66 patients were using PAP treatment. 24 (36.3\%) patients were excluded because the duration of PAP treatment did not exceed 6 months during the study period. Finally, 42 participants were analyzed for 6-month PAP adherence. Over 6 months, 32 patients showed good adherence, and 10 showed poor adherence, resulting in total PAP adherence of $76.1 \%$ after 6 months (Table 2). The mean resid-

Table 1. Demographic characteristics of the study population

\begin{tabular}{lc}
\hline \multicolumn{1}{c}{ Characteristics } & Values $(\mathrm{n}=66)$ \\
\hline Age, year & $55.7 \pm 13.5$ \\
Sex, $\mathrm{n}(\%)$ & $50(75.7)$ \\
$\quad$ Male & $16(24.2)$ \\
$\quad$ Female & $166.8 \pm 9.4$ \\
Height, cm & $75.2 \pm 17.4$ \\
Weight, kg & $26.9 \pm 5.4$ \\
BMI, kg/m ${ }^{2}$ & \\
Polysomnography data & $336.4 \pm 188.6$ \\
$\quad$ Snoring index (events/hour) & $35.2 \pm 20.3$ \\
AHI (events/hour) & $78.3 \pm 12.6$ \\
Minimum $\mathrm{O}_{2}$ saturation, \% & $92.4 \pm 4.5$ \\
Average $\mathrm{O}_{2}$ saturation, \% & \\
\hline
\end{tabular}

Data are presented as mean \pm standard deviation unless otherwise indicated. AHI: apnea-hypopnea index, BMI: body mass index ual AHI of good adherence group was 4.5.

No significant correlation with good adherence was found for age, sex, diabetes mellitus (DM), heart disease, height, weight, BMI, systolic blood pressure (SBP), or diastolic blood pressure (DBP). But hypertension (HTN) was significantly correlated with poor PAP adherence.

Additionally, we analyzed the predictors of PAP adherence, based on PSG data (Table 2). Rapid eye movement (REM) sleep latency, the AHI of non-REM (NREM) sleep, the AHI of supine position, the total arousal index, and the respiratory arousal index were significantly correlated with good PAP treatment adherence. However, total sleep time, NREM sleep time, REM sleep time, sleep latency, sleep efficiency, wake after sleep onset (WASO), AHI of REM sleep, and AHI of non-supine position were not significantly correlated with good adherence to PAP treatment.

\section{Adherence based on 3 months}

During 2019, 66 participants were prescribed PAP treatment, of which 56 participants has maintained PAP treatment for more than 3 months (Table 3). Therefore, the 3-month adherence rate was found to be $84.8 \%$, their mean residual AHI was 2.9.

Poor adherence of PAP treatment for 3 months was significantly correlated with hypertension, which was similar with the findings for 6 months results. However, no significant correlations were found with age, sex, DM, heart disease, height, weight, BMI, SBP, and DBP.

Table 3 also shows the results of examining factors associated with PAP adherence based on the PSG data. Similar to the 6-month results, REM sleep latency, AHI of NREM sleep, and AHI of supine position showed significant correlations with good adherence. In contrast with the 6-month results, respiratory arousal index and total arousal index were not correlated with 3-month PAP adherence. No significant differences were observed between the two groups for total sleep time, NREM sleep time, REM sleep time, sleep latency, sleep efficiency, WASO, AHI of REM sleep and AHI of non-supine position.

\section{Discussion}

The present study showed a 6-month PAP adherence of 76.1\% and 3-month PAP adherence of $84.8 \%$ at a regional, tertiary sleep center in Korea. This is the first study reporting the adherence of PAP treatment in patients with OSA since PAP was included to NHI service in Korea.

These results are much higher than those reported by previous studies in Korea. Kim et al. reported a 35.7\% adherence rate after 6 months, in 2015, and another study group reported a 59.4\% adherence rate for PAP treatment after 3 months. ${ }^{10-12}$ 
Table 2. Comparison of the characteristics between the good and poor adherence groups for 6 months

\begin{tabular}{|c|c|c|c|}
\hline Variables & Good adherence $(n=32)$ & Poor adherence $(n=10)$ & $p$ \\
\hline Age, year & $54.3 \pm 12.8$ & $61.0 \pm 11.2$ & 0.209 \\
\hline Sex, n (\%) & & & 0.105 \\
\hline Male & $27(84.4)$ & $6(60)$ & \\
\hline Female & $5(15.6)$ & $4(40.0)$ & \\
\hline Hypertension, n (\%) & $12(37.5)$ & $8(80.0)$ & $0.031^{*}$ \\
\hline DM, n (\%) & $4(12.5)$ & $3(30.0)$ & 0.236 \\
\hline Heart disease, $\mathrm{n}(\%)$ & $2(6.3)$ & $0(0.0)$ & 0.408 \\
\hline Height, cm & $169.4 \pm 9.1$ & $163.5 \pm 7.9$ & 0.087 \\
\hline Weight, kg & $76.9 \pm 17.6$ & $71.2 \pm 23.6$ & 0.160 \\
\hline $\mathrm{BMI}, \mathrm{kg} / \mathrm{m}^{2}$ & $26.6 \pm 4.6$ & $26.5 \pm 7.8$ & 0.658 \\
\hline SBP, mm Hg & $134.0 \pm 16.1$ & $136.2 \pm 18.1$ & 0.701 \\
\hline DBP, mm Hg & $84.9 \pm 8.9$ & $81.8 \pm 9.7$ & 0.296 \\
\hline \multicolumn{4}{|l|}{ Polysomnography data } \\
\hline Total sleep time, min & $330.7 \pm 60.5$ & $343.7 \pm 57.7$ & 0.679 \\
\hline NREM sleep time & $286.0 \pm 52.6$ & $289.5 \pm 62.0$ & 0.779 \\
\hline REM sleep time & $44.7 \pm 24.4$ & $54.2 \pm 25.1$ & 0.184 \\
\hline Sleep latency, $\min$ & $22.0 \pm 21.6$ & $18.5 \pm 13.8$ & 0.976 \\
\hline REM sleep latency, min & $141.6 \pm 85.8$ & $84.2 \pm 57.8$ & $0.022^{*}$ \\
\hline Sleep efficiency, \% & $77.3 \pm 13.8$ & $80.6 \pm 8.7$ & 0.690 \\
\hline WASO, min & $75.3 \pm 61.2$ & $63.9 \pm 34.0$ & 0.988 \\
\hline AHI (event/hr) & $36.4 \pm 18.1$ & $25.4 \pm 20.3$ & 0.059 \\
\hline NREM AHI & $36.5 \pm 18.7$ & $23.7 \pm 22.4$ & $0.022 *$ \\
\hline REM AHI & $33.8 \pm 21.4$ & $28.7 \pm 18.3$ & 0.555 \\
\hline Non-supine AHI & $19.2 \pm 23.8$ & $16.7 \pm 33.9$ & 0.413 \\
\hline Supine AHI & $40.2 \pm 19.5$ & $27.2 \pm 11.3$ & $0.030^{*}$ \\
\hline Total arousal index (arousal/hr) & $35.2 \pm 22.3$ & $23.4 \pm 19.3$ & $0.024^{*}$ \\
\hline Respiratory arousal index & $26.5 \pm 18.3$ & $16.5 \pm 20.7$ & $0.036^{*}$ \\
\hline \multicolumn{4}{|l|}{ Sleep stage } \\
\hline $\mathrm{N} 1$ (min) & 117.9 & 99.3 & 0.072 \\
\hline $\mathrm{N} 2(\min )$ & 154.4 & 170.4 & 0.294 \\
\hline $\mathrm{N} 3(\mathrm{~min})$ & 13.6 & 19.8 & 0.199 \\
\hline REM (min) & 44.7 & 54.2 & 0.184 \\
\hline
\end{tabular}

Data are presented as mean \pm standard deviation unless otherwise indicated. *statistically significant $(p<0.05)$. DM: diabetes mellitus, BMI: body mass index, SBP: systolic blood pressure, DBP: diastolic blood pressure, REM: rapid eye movement, WASO: wake after sleep onset, NREM: non-rapid eye movement, AHI: apnea-hypopnea index

Similar to the findings of previous studies in Korea, many studies have explored PAP adherence among OSA patients in worldwide, and the results have varied. Up to $30 \%$ of patients fail to initiate therapy after diagnosis, and among those who start therapy, approximately $25 \%$ stop within the first year, and fewer than $50 \%$ remain adherent in the long term. ${ }^{13,14}$

In Korea, a single universal health insurance system provides insurance for over $99 \%$ of the population. ${ }^{8}$ Starting in July 2018, patients with OSA have begun to receive benefits from the National Health Insurance Service (NHIS). Because
PAP treatment requires comparatively expensive equipment, the financial burden is closely related to compliance, and national policies, such as the health insurance system, can affect PAP treatment compliance. ${ }^{15}$ Therefore, authors assessed that the high compliance of PAP treatment in this study compared to previous may be due to the benefits provided by the NHIS from 2018 in Korea. In addition to economic variables, adherence to PAP treatment is affected by various device-related factors and side effects, psychological factors, family, and spouserelated factors, socioeconomic factors, and physician-related 
Table 3. Comparison of the characteristics between the good and poor adherence groups for 3 months

\begin{tabular}{|c|c|c|c|}
\hline Variables & Good adherence $(n=56)$ & Poor adherence $(n=10)$ & $p$ \\
\hline Age, year & $54.9 \pm 13.9$ & $61.0 \pm 11.2$ & 0.260 \\
\hline Sex, n (\%) & & & 0.210 \\
\hline Male & $44(78.6)$ & $6(60.0)$ & \\
\hline Female & $12(21.4)$ & $4(40.0)$ & \\
\hline Hypertension, n (\%) & $19(33.9)$ & $8(80)$ & $0.010^{*}$ \\
\hline $\mathrm{DM}, \mathrm{n}(\%)$ & $8(14.3)$ & $3(30)$ & 0.259 \\
\hline Heart disease, $\mathrm{n}(\%)$ & $4(7.1)$ & $0(0)$ & 0.373 \\
\hline Height, $\mathrm{cm}$ & $167.5 \pm 9.7$ & $163.5 \pm 8.0$ & 0.184 \\
\hline Weight, kg & $76.0 \pm 16.3$ & $71.2 \pm 23.7$ & 0.170 \\
\hline BMI, $\mathrm{kg} / \mathrm{m}^{2}$ & $27.1 \pm 5.0$ & $26.5 \pm 7.8$ & 0.507 \\
\hline SBP, mm Hg & $133.0 \pm 16.1$ & $136.3 \pm 18.2$ & 0.523 \\
\hline DBP, mm Hg & $83.9 \pm 8.3$ & $81.8 \pm 9.7$ & 0.444 \\
\hline \multicolumn{4}{|l|}{ Polysomnography data } \\
\hline Total sleep time, min & $326.5 \pm 61.2$ & $343.7 \pm 57.8$ & 0.707 \\
\hline NREM sleep time & $279.5 \pm 53.0$ & $289.5 \pm 62.0$ & 0.986 \\
\hline REM sleep time & $47.2 \pm 24.2$ & $54.2 \pm 25.2$ & 0.304 \\
\hline Sleep latency, min & $21.3 \pm 21.0$ & $18.5 \pm 13.9$ & 0.830 \\
\hline REM sleep latency & $133.0 \pm 81.0$ & $84.2 \pm 57.8$ & $0.022 *$ \\
\hline Sleep efficiency, \% & $77.1 \pm 14.1$ & $80.6 \pm 8.7$ & 0.674 \\
\hline WASO, min & $77.1 \pm 57.8$ & $63.9 \pm 34.1$ & 0.809 \\
\hline AHI (event/hr) & $37.0 \pm 20.0$ & $25.4 \pm 20.3$ & 0.060 \\
\hline NREM AHI & $37.7 \pm 20.5$ & $23.8 \pm 22.4$ & $0.024^{*}$ \\
\hline REM AHI & $33.4 \pm 23.7$ & $28.8 \pm 18.4$ & 0.707 \\
\hline Non-supine AHI & $20.1 \pm 24.7$ & $16.8 \pm 34.0$ & 0.415 \\
\hline Supine AHI & $41.3 \pm 22.0$ & $27.2 \pm 11.4$ & $0.036^{*}$ \\
\hline Total arousal index (arousal/hr) & $32.0 \pm 20.4$ & $23.4 \pm 19.4$ & 0.052 \\
\hline Respiratory arousal index & $24.3 \pm 18.2$ & $16.5 \pm 20.7$ & 0.059 \\
\hline \multicolumn{4}{|l|}{ Sleep stage } \\
\hline $\mathrm{N} 1(\min )$ & 102.5 & 99.3 & 0.283 \\
\hline $\mathrm{N} 2(\min )$ & 161.4 & 170.4 & 0.491 \\
\hline $\mathrm{N} 3(\min )$ & 15.3 & 19.8 & 0.249 \\
\hline $\operatorname{REM}(\min )$ & 47.2 & 54.2 & 0.304 \\
\hline
\end{tabular}

Data are presented as mean \pm standard deviation unless otherwise indicated. *statistically significant $(p<0.05)$. DM: diabetes mellitus, BMI: body mass index, SBP: systolic blood pressure, DBP: diastolic blood pressure, REM: rapid eye movement, WASO: wake after sleep onset, NREM: non-rapid eye movement, AHI: apnea-hypopnea index

factors. Device-related factors include discomfort caused by high pressure, vague discomfort of the device user, social and personal prejudices against device users, social and personal prejudices against the machine itself, which can lead patients to avoid the use of a troublesome system. Additional research focused on these factors should be included in a future study.

Patients with OSA often have one or more comorbidities, including obesity, dyslipidemia, glucose intolerance, and HTN. ${ }^{16,17}$ With obesity, periods of obstructed breathing can result in profound intermittent hypoxia, with hypercapnia, negative intra- thoracic pressure swings, and arousals. ${ }^{18}$ Physiological changes that occur in response to obstructed breathing can include sympathetic activation, inflammation, oxidative stress, and endothelial dysfunction. ${ }^{19}$ As a result, this series of diseases can result in increased blood pressure and HTN. ${ }^{16}$ In studies examining the prevalence of sleep apnea amongst HTN populations, $20-40 \%$ of individuals have been diagnosed with comorbid sleep apnea, in our study, 40\% of participants with OSA also had HTN, and patients with comorbid HTN showed a significant correlation with poor adherence. Future trials should 
explore how HTN comorbidity affects poor adherence to PAP treatment.

We demonstrated that PSG features significantly contributed to good PAP adherence, including prolonged REM latency, higher NREM AHI, higher supine AHI, and frequent arousal. These factors imply poor sleep quality. Although no significant difference in AHI was observed between the two groups, higher adherence may be correlated with the subjective discomfort caused by poor sleep quality, resulting in patients attempting to improve compliance. Additional research focused on these subjective factors should be included in a future study.

Most obstructive sleep apnea is worse during REM sleep than during NREM. However, several studies showed increased NREM AHI compared with REM AHI with OSA, which is similar to ours that NREM AHI was higher than REM AHI (35.5 events/hr vs. 32.6 events/hr, respectively, $\mathrm{n}=66$ ). ${ }^{20} \mathrm{We}$ assessed that lower AHI during REM sleep may be due to the deprivation of REM sleep opportunities, as a result of frequent awakening. $^{20,21}$

This study has several limitations. This study was a retrospective and single-center study, with a small sample. However, no studies have yet examined effect on adherence PAP treatment when the cost of treatment is covered by NHI in Korea. Therefore, this study demonstrated that health care services that relieve the economic burden of PAP machines may have a positive effect on compliance. We believe that these results will represent a useful reference for increasing adherence in other countries where public health services are well guaranteed. Another limitation is the lack of subjective symptom evaluations, such as the use of a sleep questionnaire. A self-reported questionnaire can provide information about the participant's complaints regarding poor sleep quality. Further study remains necessary to evaluate the daytime sleepiness scale and additional data that may affect PAP adherence.

In conclusion, we demonstrated a 6-month PAP adherence was $76.1 \%$ and 3-month PAP adherence was $84.8 \%$ in patients with OSA in Korea, which showed increased adherence compared to that of previous studies conducted when PAP treatment was not insured. And authors also determined that prolonged REM latency, higher NREM AHI, and higher supine AHI are significant contributors to good PAP adherence, whereas comorbid hypertension is a factor for poor PAP compliance.

\section{Acknowledgments}

This research did not receive any specific grant from funding agencies in the public, commercial, or not-for-profit sectors.

\section{Conflicts of Interest}

The authors have no potential conflicts of interest to disclose.

\section{ORCID iDs}

Hyo-Eun Yoon

Chang-Jin Jeon

Jaechun Hwang

Ho-Won Lee

Ji-Ye Jeon https://orcid.org/0000-0002-0369-0882 https://orcid.org/0000-0003-0492-6735 https://orcid.org/0000-0002-8917-3439 https://orcid.org/0000-0002-8849-920X https://orcid.org/0000-0003-1111-3521

\section{Author Contributions}

Conceptualization: Hyo-Eun Yoon, Ji-Ye Jeon. Data curation: Hyo-Eun Yoon, Chang-Jin Jeon. Formal analysis: Hyo-Eun Yoon, Ji-Ye Jeon. Investigations: Jaechun Hwang, Ho-Won Lee. Methodology: Ji-Ye Jeon. Supervision: Chang-Jin Jeon, Ho-Won Lee. Writing-original draft: Hyo-Eun Yoon. Writing_-review \& editing: Jaechun Hwang, Ji-Ye Jeon.

\section{REFERENCES}

1. Redline S, Young T. Epidemiology and natural history of obstructive sleep apnea. Ear Nose Throat J 1993;72:20-26.

2. Nieto FJ, Young TB, Lind BK, et al. Association of sleep-disordered breathing, sleep apnea, and hypertension in a large community-based study. Sleep Heart Health Study. JAMA 2000;283:1829-1836. https:// doi.org/10.1001/jama.283.14.1829.

3. Hung J, Whitford EG, Parsons RW, Hillman DR. Association of sleep apnoea with myocardial infarction in men. Lancet 1990;336:261-264. https://doi.org/10.1016/0140-6736(90)91799-g.

4. Shahar E, Whitney CW, Redline S, et al. Sleep-disordered breathing and cardiovascular disease: cross-sectional results of the Sleep Heart Health Study. Am J Respir Crit Care Med 2001;163:19-25. https://doi. org/10.1164/ajrccm.163.1.2001008.

5. Young T, Finn L, Peppard PE, et al. Sleep disordered breathing and mortality: eighteen-year follow-up of the Wisconsin Sleep cohort. Sleep 2008;31:1071-1078. https://doi.org/10.5665/sleep/31.8.1071.

6. Patil SP, Ayappa IA, Caples SM, Kimoff RJ, Patel SR, Harrod CG. Treatment of adult obstructive sleep apnea with positive airway pressure: an American Academy of Sleep Medicine systematic review, meta-analysis, and GRADE assessment. J Clin Sleep Med 2019;15:301334. https://doi.org/10.5664/jcsm.7638.

7. Yetkin O, Kunter E, Gunen H. CPAP compliance in patients with obstructive sleep apnea syndrome. Sleep Breath 2008;12:365-367. https:// doi.org/10.1007/s11325-008-0188-4.

8. Seong SC, Kim YY, Khang YH, et al. Data resource profile: the national health information database of the National Health Insurance Service in South Korea. Int J Epidemiol 2017;46:799-800. https://doi. org/10.1093/ije/dyw253.

9. Berry RB, Budhiraja R, Gottlieb DJ, et al. Rules for scoring respiratory events in sleep: update of the 2007 AASM manual for the scoring of sleep and associated events. Deliberations of the sleep apnea definitions task force of the American Academy of Sleep Medicine. J Clin Sleep Med 2012;8:597-619. https://doi.org/10.5664/jcsm.2172.

10. Kim MJ, Kim MJ, Bae SH, Park CH, Kim DK. Predictors of adherence with positive airway pressure treatment in patients with obstructive sleep apnea in Korean. J Rhinol 2015;22:89-95. https://doi.org/10.18787/ jr.2015.22.2.89.

11. Han EK, Yoon IY, Chung SH. The compliance and effect of CPAP in obstructive sleep apnea syndrome. Sleep Med Psychophysiol 2006;13: 52-58.

12. Choi JB, Lee SH, Jeong DU. Compliance of nasal continuous positive airway pressure in patients with obstructive sleep apnea syndrome. Sleep Med Psychophysiol 2006;13:27-32.

13. Lettieri CJ, Walter RJ. Group education on CPAP--a response. J Clin Sleep Med 2013;9:975-976. https://doi.org/10.5664/jcsm.3012.

14. Means MK, Ulmer CS, Edinger JD. Ethnic differences in continuous positive airway pressure (CPAP) adherence in veterans with and without psychiatric disorders. Behav Sleep Med 2010;8:260-273. https://doi. 
org/10.1080/15402002.2010.509255.

15. Brin YS, Reuveni H, Greenberg S, Tal A, Tarasiuk A. Determinants affecting initiation of continuous positive airway pressure treatment. Isr Med Assoc J 2005;7:13-18.

16. Phillips CL, O'Driscoll DM. Hypertension and obstructive sleep apnea. Nat Sci Sleep 2013;5:43-52. https://doi.org/10.2147/NSS.S34841.

17. Phillips CL, Yee BJ, Marshall NS, Liu PY, Sullivan DR, Grunstein RR. Continuous positive airway pressure reduces postprandial lipidemia in obstructive sleep apnea: a randomized, placebo-controlled crossover trial. Am J Respir Crit Care Med 2011;184:355-361. https://doi.org/10.1164/ rccm.201102-0316OC.
18. Kasai T, Floras JS, Bradley TD. Sleep apnea and cardiovascular disease: a bidirectional relationship. Circulation 2012;126:1495-1510. https:// doi.org/10.1161/CIRCULATIONAHA.111.070813.

19. McNicholas WT. Obstructive sleep apnea and inflammation. Prog Cardiovasc Dis 2009;51:392-399. https://doi.org/10.1016/j.pcad.2008.10.005.

20. Siddiqui F, Walters AS, Goldstein D, Lahey M, Desai H. Half of patients with obstructive sleep apnea have a higher NREM AHI than REM AHI. Sleep Med 2006;7:281-285. https://doi.org/10.1016/j.sleep.2005.10.006.

21. Mador MJ, Kufel TJ, Magalang UJ, Rajesh SK, Watwe V, Grant BJ. Prevalence of positional sleep apnea in patients undergoing polysomnography. Chest 2005;128:2130-2137. https://doi.org/10.1378/chest.128.4.2130. 